\title{
Surface-Plasmon-Induced Modification on the Spontaneous Emission Spectrum via Subwavelength-Confined Anisotropic Purcell Factor
}

\author{
Ying Gu, ${ }^{* \dagger}{ }^{\dagger}$ Luojia Wang, ${ }^{\dagger}$ Pan Ren, ${ }^{\dagger}$ Junxiang Zhang, ${ }^{\ddagger}$ Tiancai Zhang, ${ }^{\ddagger}$ Olivier J. F. Martin, ${ }^{\S}$
} and Qihuang Gong*, $\dagger$

\author{
${ }^{\dagger}$ State Key Laboratory for Mesoscopic Physics, Department of Physics, Peking University, Beijing 100871, China \\ ${ }^{\ddagger}$ State Key Laboratory of Quantum Optics and Quantum Optics Devices, Institute of Opto-Electronics, Shanxi University, Taiyuan \\ 030006, China \\ ${ }^{\S}$ Nanophotonics and Metrology Laboratory, Swiss Federal Institute of Technology Lausanne (EPFL), EPFL-STI-NAM, ELG Station \\ 11, CH-1015 Lausanne, Switzerland
}

Supporting Information

ABSTRACT: The mechanism of using the anisotropic Purcell factor to control the spontaneous emission linewidths in a four-level atom is theoretically demonstrated; if the polarization angle bisector of the two dipole moments lies along the axis of large/small Purcell factor, destructive/constructive interference narrows/widens the fluorescence center spectral lines. Large anisotropy of the Purcell factor, confined in the subwavelength optical mode volume, leads to rapid spectral line narrowing of atom approaching a metallic nanowire, nanoscale line width pulsing following periodically varying decay rates near a periodic metallic nanostructure, and dramatic modification on the spontaneous emission spectrum near a custom-designed resonant plasmon nanostructure. The combined system opens a good perspective for applications in ultracompact active quantum devices.

KEYWORDS: Surface plasmon, spontaneous emission, Purcell factor, quantum emitter, quantum interference

$\mathrm{R}$ ecent developments in nanotechnology and information technologies have made nanoscale light-matter interaction a tremendous research focus. ${ }^{1}$ Small optical mode area in nanofiber-based photonic structures plays a significant role allowing low-light level quantum optical phenomena, such as electromagnetically induced transparency in the nanowatt regime, ${ }^{2,3}$ four wave mixing with great gain, ${ }^{4}$ and two-photon absorption with sharp peaks in the Rubidium vapor. ${ }^{5}$ Ultrasmall optical mode volume in plasmon nanostructures ${ }^{6}$ leads to strong coupling between surface plasmons and quantum emitters, which enables the vacuum Rabi splitting, ${ }^{7,8}$ the Fano lineshapes in the absorption spectrum, ${ }^{9-12}$ and its obvious influence on the two-photon statistics. ${ }^{13}$

Superior to many available photonic nanostructures, associated with ultrasmall optical mode volume, ${ }^{6}$ plasmonic structures present the key advantage of a large subwavelengthconfined anisotropic vacuum, that is, large anisotropic Purcell factor, ${ }^{14,15}$ which originates from an anisotropic electric mode density of collective oscillations of free electrons in metals. ${ }^{16-19}$ Another advantage is strong evanescent field of metallic nanostructures, which has promoted many applications, for example, SERS, ${ }^{20}$ nanometer biosensors and waveguides, ${ }^{21}$ nonlinear optical frequency mixing, ${ }^{22-24}$ solar cell, ${ }^{25,26}$ and so forth. Through modifying the population of excited states and decay rate of quantum emitters near plasmon structure, the fluorescence enhancement and quenching of fluorescent

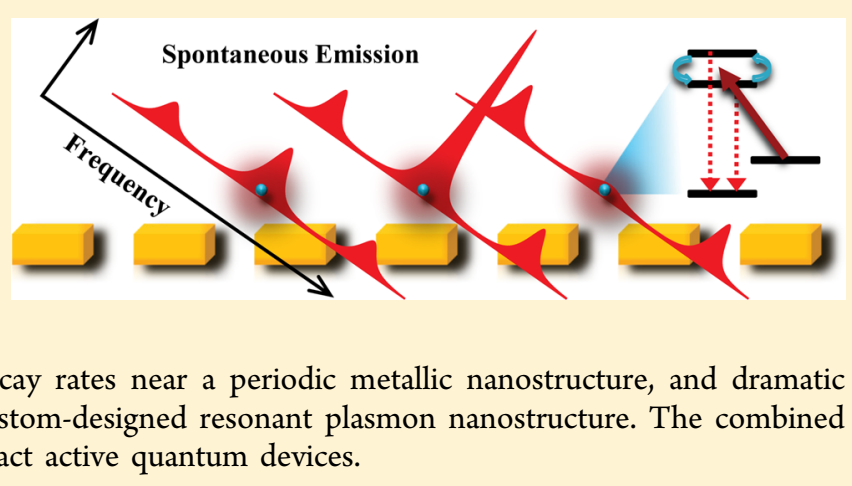

molecules and semiconductor quantum dots can be controlled well. $^{27-32}$ By confining the light into nanoscale volumes, plasmonic elements allow for a nanoscale realization of Mollow triplet of emission spectra and antibunching of emission photons of single molecules that traditional technique can not be accessible. ${ }^{1,33,34}$ Through the nanoscale coupling between the surface plasmon modes and single quantum emitter, the directional and efficiency generation of single photons ${ }^{17-19}$ and entanglement of two qubits ${ }^{35}$ were proposed. These advantages, thanks to rapidly developed fabrication techniques, make the plasmonic nanostructure a promising candidate for applications in ultracompact active quantum devices, for example, single photons sources ${ }^{17-19}$ and atomic spectroscopy on a chip. ${ }^{36}$

Crossing damping between two closely lying upper states of atoms, originated from the interaction with the common vacuum of electromagnetic fields, has led to ultranarrow spectral line in spontaneous emission, ${ }^{37,38}$ which is potentially of interest in spectroscopy. Except for nearly parallel dipoles, such interesting interference phenomenon can not occur in a vacuum due to small or zero value of crossing damping terms. A natural atom generally has arbitrary polarized dipoles, so these

Received: February 16, 2012

Revised: April 8, 2012

Published: April 18, 2012 
effects are scarcely observed experimentally. Anisotropic Purcell factor can provide nonzero or large crossing damping even with orthogonal dipoles. Hence various photonic structures, for example, photonic band gap structures, ${ }^{39,40}$ layered dielectric waveguides, ${ }^{41}$ left-handed material, ${ }^{42}$ and plasmonic structures, ${ }^{43}$ were proposed to enhance this interference effect via the anisotropic Purcell factor. ${ }^{44}$ However, the mechanism underlying the anisotropic Purcell factor control of the spectral lines associated with spontaneous emissions remains unclear.

To address this issue, in this Letter we begin by theoretically resolving the underlying mechanism, specifically concentrating on four-level atoms with closely lying upper levels. As illustrated in Figure 1, if the angle bisector of two arbitrarily

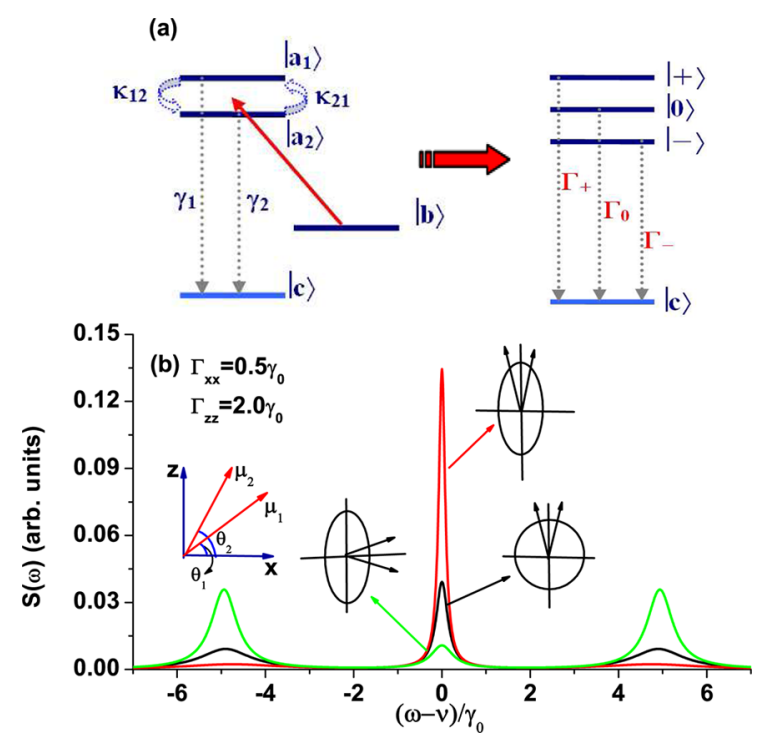

Figure 1. (a) The schematics of a four-level atomic system and its dressed states. (b) Narrowing (red curve) and broadening (green curve) of the spectral lines associated with spontaneous emission via anisotropic decay rates, compared with lines (black curve) for a vacuum. Two dipole moments lie at an angle of $0.2 \pi$. Rabi frequencies are $\Omega_{1}=\Omega_{2}=3.5 \gamma_{0}$.

polarized dipole moments lies along the major/minor axis of the effective Purcell factor ellipse, destructive/constructive interference narrows/widens the center spectral lines of fluorescence. Using the mechanism, we study the quantum interference behavior in the spontaneous emission spectrum via the subwavelength-confined anisotropic Purcell factor: atomic spectral line rapid narrowing near a metallic nanowire, nanoscale line width pulsing, that is, periodically from narrowing to widening, near a periodic metallic nanostructure, and dramatic modification, even subnatural line width for two orthogonal dipoles, of spontaneous emission near a customdesigned resonant plasmon nanostructure. These results lay the foundation of the light-atom interaction within the ultrasmall optical mode area. Also, the combined system of atoms and the metallic nanostucture may be integrated into ultracompact active quantum devices.

Mechanism of the Spontaneous Emission Spectrum Control. Consider a closed four-level atomic system with two closely lying upper levels $\left|a_{1}\right\rangle$ and $\left|a_{2}\right\rangle$ and two ground states $|b\rangle$ and $|c\rangle$ (Figure 1a). The transitions $\left|a_{1}\right\rangle \leftrightarrow|b\rangle$ and $\left|a_{2}\right\rangle \leftrightarrow|b\rangle$ are driven by a pump field with frequency $\nu$ and Rabi frequencies $\Omega_{1}$ and $\Omega_{2}$, and the detunings $\Delta_{1}=\omega_{a 1}-\nu$ and $\Delta_{2}$ $=\omega_{a 2}-\nu$ where $\omega_{a 1}-\omega_{a 2}=\omega_{12}$. In the dipole, rotating-wave and Weisskopf-Wigner approximations, the state vectors $A_{1}(t)$, $A_{2}(t)$, and $B(t)$ obey the Schrodinger equations ${ }^{45-47}$

$$
\begin{aligned}
& \frac{\mathrm{d}}{\mathrm{d} t} A_{1}(t)=-\frac{\gamma_{1}}{2} A_{1}(t)-\frac{\kappa_{12}}{2} A_{2}(t) e^{i \omega_{12} t}+\Omega_{1} e^{i \Delta_{1} t} B(t) \\
& \frac{\mathrm{d}}{\mathrm{d} t} A_{2}(t)=-\frac{\gamma_{2}}{2} A_{2}(t)-\frac{\kappa_{21}}{2} A_{1}(t) e^{-i \omega_{12} t}+\Omega_{2} e^{i \Delta_{2} t} B(t) \\
& \frac{\mathrm{d}}{\mathrm{d} t} B(t)=-\Omega_{1}^{*} e^{-i \Delta_{1} t} A_{1}(t)-\Omega_{2}^{*} e^{-i \Delta_{2} t} A_{2}(t)
\end{aligned}
$$

where $\gamma_{1}$ and $\gamma_{2}$ are decay rates from $\left|a_{1}\right\rangle$ and $\left|a_{2}\right\rangle$ to $|c\rangle$, and $\kappa_{12}$ and $\kappa_{21}$ are crossing damping terms between $\left|a_{1}\right\rangle$ and $\left|a_{2}\right\rangle$, respectively. For the closing states $\left|a_{1}\right\rangle$ and $\left|a_{2}\right\rangle, \kappa_{12}=\kappa_{21}=\kappa$. If $y$-axis is allowed to be the quantum axis and the values of dipole moments $\mu_{1}=\mu_{2}=\mu$, and $\theta_{1,2}$ to be the intersection angles between $\vec{\mu}_{1,2}$ and $x$-axis, in the anisotropic vacuum, $\gamma_{1,2}=\Gamma_{x x}$ $\cos ^{2} \theta_{1,2}+\Gamma_{z z} \sin ^{2} \theta_{1,2}$ and $\kappa=\Gamma_{x x} \cos \theta_{1} \cos \theta_{2}+\Gamma_{z z} \sin \theta_{1} \sin$ $\theta_{2}$, where $\Gamma_{z z} / \gamma_{0}=3 \lambda_{a c} \operatorname{Im} G_{z z}, \Gamma_{x x} / \gamma_{0}=3 \lambda_{a c} \operatorname{Im} G_{x x}, \gamma_{0}\left(=\mu^{2} \omega_{a c}^{3}\right)$ $\left.\left(3 \pi \varepsilon_{0} \hbar c_{3}\right)\right)$ is the decay rate in a vacuum, and $G_{\beta \beta}$ with $\beta=x, y, z$ are the Green's tensor coefficients. ${ }^{33,48}$ In the following, we will see that the $x$ - and $z$-direction have different Purcell factors ${ }^{14}$ $\Gamma_{x x} / \gamma_{0}$ and $\Gamma_{x x} / \gamma_{0}$, which has guaranteed the quantum interference effects of spectral line width narrowing and widening of spontaneous emission. The spontaneous emission spectra $S(\omega)=(1 / \pi) \operatorname{Re} \int_{0}^{\infty} \mathrm{d} \tau\left\langle E^{-}(t) E^{+}(t+\tau)\right\rangle e^{i \omega \tau}$ for $t \rightarrow \infty$, where $E^{-}(t)$ is the field operator of emission photons from the upper levels to $|c\rangle$, were investigated in the framework of quantum regression theorem. ${ }^{45-47}$

We use the dressed state analysis ${ }^{49}$ to develop a mechanism that controls the spectral linewidths of spontaneous emission via the anisotropic Purcell factor (Figure 1a). Writing the interaction Hamiltonian of the system as

$$
\begin{aligned}
H= & \hbar \Delta_{1}\left|a_{1}\right\rangle\left\langle a_{1}\left|+\hbar \Delta_{2}\right| a_{2}\right\rangle\left\langle a_{2}\right| \\
& +\left(\hbar \Omega_{1}\left|a_{1}\right\rangle\left\langle b\left|+\hbar \Omega_{2}\right| a_{2}\right\rangle\langle b|+\text { H. c. }\right)
\end{aligned}
$$

then taking $\Omega_{1}=\Omega_{2}=\Omega$ and $\Delta_{1}=\omega_{12} / 2$, we obtain expressions for the eigenvalues and eigenvectors

$$
\lambda_{+}=\frac{\Omega_{\mathrm{R}}}{2}, \quad \lambda_{0}=0, \quad \lambda_{-}=-\frac{\Omega_{\mathrm{R}}}{2}
$$

and

$$
\left(\begin{array}{c}
|+\rangle \\
|0\rangle \\
|-\rangle
\end{array}\right)=\left(\begin{array}{ccc}
\frac{1+\varepsilon}{2} & 2 \eta & \frac{1-\varepsilon}{2} \\
-2 \eta & \varepsilon & 2 \eta \\
-\frac{1-\varepsilon}{2} & 2 \eta & -\frac{1+\varepsilon}{2}
\end{array}\right)\left(\begin{array}{c}
\left|a_{1}\right\rangle \\
|b\rangle \\
\left|a_{2}\right\rangle
\end{array}\right)
$$

where $\Omega_{\mathrm{R}}^{2}=\omega_{12}^{2}+8 \Omega^{2}, \varepsilon=\omega_{12} / \Omega_{\mathrm{R}}$, and $\eta=\Omega / \Omega_{\mathrm{R}}$. The equations of motion for the density matrices of dressed states are given in the Supporting Information. The modified decay rates of their diagonal matrix elements, $\rho_{00}$ and $\rho_{++}$, are given by the expressions

$$
\begin{aligned}
& \Gamma_{0}=\left(\gamma_{1}+\gamma_{2}-2 \kappa\right) 4 \eta^{2} \\
& \Gamma_{ \pm}=\left(\gamma_{1}+\gamma_{2}\right) \frac{1+\varepsilon^{2}}{4} \pm\left(\gamma_{1}-\gamma_{2}\right) \frac{\varepsilon}{2}+\kappa \frac{1-\varepsilon^{2}}{2}
\end{aligned}
$$

which determine separately the linewidths of the central peak and the emission spectrum sidebands. If two dipoles are 
parallel, then $\Gamma_{0}$ is zero, which means the trapping condition for spontaneous emission cancellation ${ }^{45}$ cannot be broken whether the vacuum be isotropic or not. ${ }^{50}$ If $\omega_{12}^{2} \ll 8 \Omega^{2}, \varepsilon$ can be ignored and $4 \eta^{2} \doteq 1 / 2$, then $\Gamma_{0} \doteq\left(\gamma_{1}+\gamma_{2}\right) / 2-\kappa$ and $\Gamma_{ \pm} \doteq\left(\gamma_{1}\right.$ $\left.+\gamma_{2}\right) / 4+\kappa / 2$

The anisotropic Purcell factor can be described by an effective decay rate ellipse (see the inset in Figure 1c), where $\sqrt{ } \Gamma_{z z}$ determines the length of the major axis and $\sqrt{\Gamma_{x x}}$ the minor axis. From the dressed state analysis, we find that when the angle bisector of two dipole moments with arbitrary polarization is along the major axis of the effective decay rate ellipse, $\Gamma_{0}$ reaches its minimum; that is, destructive interference occurs and leads to maximal narrowing of the central fluorescence spectral lines. In contrast, when their angle bisector aligns with the minor axis, $\Gamma_{0}$ becomes maximal resulting in constructive interference characterized by the maximal broadening of the central lines. The origin of line width narrowing and broadening is the slow and fast decays of the central dressed population. Simultaneously, but conversely, the line width of the two sidebands becomes wider than that in a vacuum for destructive interference and vice versa for constructive interference.

The above points were clearly verified by full numerical calculations of emission spectra $S(\omega)$ represented graphically in Figure $1 \mathrm{~b}$ with parameters $\Gamma_{z z}=2.0 \gamma_{0}$ and $\Gamma_{x x}=0.5 \gamma_{0}$. Generally, the condition $\omega_{12}^{2} \ll 8 \Omega^{2}$ is fulfilled. Here we let $\omega_{12}$ $=0$. We can see that, for two dipoles with $\theta_{1}=0.4 \pi$ and $\theta_{2}=$ $0.6 \pi$, both destructive and constructive interferences have been established, that is, relative to the line width ratio $\Gamma_{0} / \Gamma_{ \pm}=$ 0.2112 in a vacuum, line width variations from narrowing $\Gamma_{0} /$ $\Gamma_{ \pm}=0.0528$ to broadening $\Gamma_{0} / \Gamma_{ \pm}=0.8446$ are observed. By enlarging the anisotropy of the Purcell factor, this variation can be further increased and, when it is sufficiently large, two arbitrary polarized dipoles can produce strong interference in spontaneous emission. This overcomes the previous trapping condition in a vacuum that ultranarrow spectral lines only occur for nearly parallel dipoles, ${ }^{37}$ which limited the experimental observation of quantum interference. Thus, by creating large anisotropic Purcell factor, these atomic interferences should be more easily observed experimentally.

The changing process of spectral line width in spontaneous emission with the intersection angle of two dipoles is investigated. Let us first see their properties in a vacuum, as shown in Figure $2 \mathrm{~b}$, when $\theta=0.0$, that is, two dipoles are parallel, spontaneous emission cancelation happens; ${ }^{45}$ when $\theta=$ $0.025 \pi$, that is, two dipoles are approximately parallel, we observe the spectral line narrowing in spontaneous emission; ${ }^{37}$ when $\theta=0.1 \pi$, though it is still a constructive interference, only a small narrowing is obtained; when $\theta=0.25 \pi, \kappa=0.0$ and not any interference exists, about which, several literature looked $\kappa$ / $\left(\gamma_{1}+\gamma_{2}\right)$ of two orthogonal dipoles as a standard of quantum interference in the case of anisotropic Purcell factor; ${ }^{39-44}$ continually increasing $\theta$ to $0.4 \pi$, an obvious destructive interference happens. For the anisotropic Purcell factor, we still take $\Gamma_{z z}=2.0, \Gamma_{x x}=0.5$. Figure 2a shows the situation that long axis of effective decay rate ellipse is the angle bisector of two dipoles. For all situations discussed above, via constructive interference induced by the anisotropic Purcell factor, their center spectral lines become narrowing while their sideband line width become widening. Especially for two orthogonal dipoles, the effective narrowing of center lines is obtained. In the contrast, when the short axis is the angle bisector of two dipoles, their center lines become wider and their sideband line

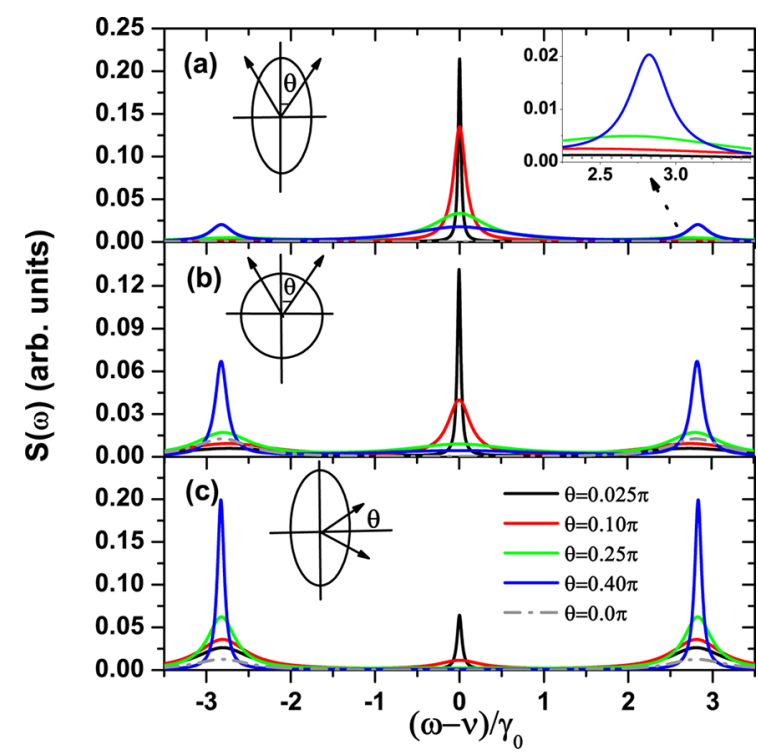

Figure 2. Spontaneous emission spectra as a function of the angle $\theta$ in anisotropic decay rates (a,c) and in a vacuum (b). $\Gamma_{x x}=0.5 \gamma_{0}, \Gamma_{z z}=$ $2.0 \gamma_{0}, \omega_{12}=0.0$, and $\Omega_{1}=\Omega_{2}=2.0 \gamma_{0}$.

width become narrower due to the destructive interference, as shown in Figure $2 \mathrm{c}$. It is worthy to point out again that for two parallel dipoles the trapping condition for spontaneous emission cancelation ${ }^{45}$ cannot be broken whenever the vacuum is isotropic or anisotropic. ${ }^{50}$ Therefore, via the anisotropic Purcell factor, arbitrary polarized dipoles can have very strong interference effects in spontaneous emission.

Now we explore the effect of the spacing of two upper levels on resonance fluorescence. Equation 6 show that, when $\varepsilon \neq 0$, the difference of decay rates in the dressed populations $\rho_{++}$and $\rho_{--}$can lead to asymmetry of two sidebands in spontaneous emission. Figure 3 displays the asymmetrical emission peaks as

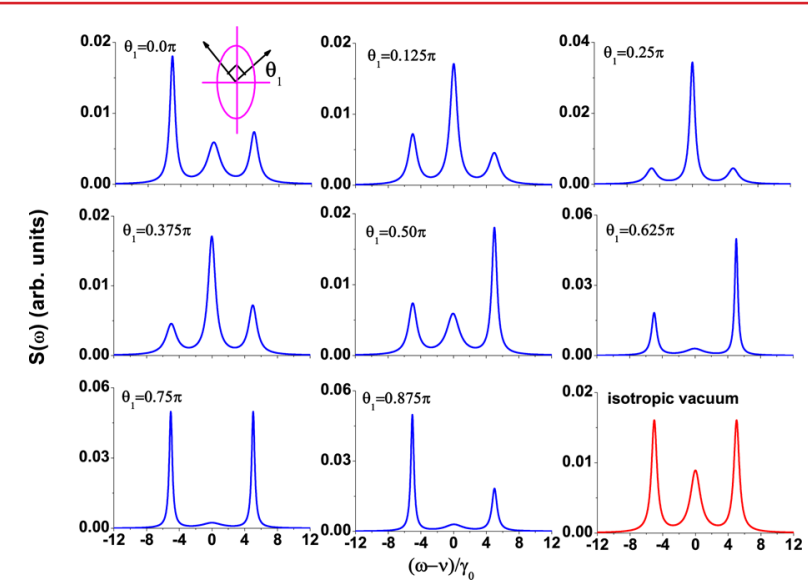

Figure 3. Spontaneous emission spectra of two orthogonal dipoles in anisotropic decay rates with $\Gamma_{x x}=0.5 \gamma_{0}, \Gamma_{z z}=2.0 \gamma_{0} . \omega_{12}=2.0 \gamma_{0}, \Omega_{1}=$ $\Omega_{2}=3.5 \gamma_{0}$.

a function of $\theta_{1}$. The parameters are $\omega_{12}=2.0 \gamma_{0}$ and $\theta_{2}-\theta_{1}=$ $0.5 \pi$. It is seen that, except for the symmetry spectra occurred at $\theta_{1}=0.25 \pi$ with maximum constructive interference and $\theta_{1}=$ $0.75 \pi$ with maximum destructive interference, other several pairs, such as $\theta_{1}=0.0 \pi$ and $\theta_{1}=0.5 \pi, \theta_{1}=0.125 \pi$ and $\theta_{1}=$ $0.375 \pi, \theta_{1}=0.625 \pi$ and $\theta_{1}=0.875 \pi$, exhibit the mirror symmetry of the emission peaks. Besides, with an increment of 
$\theta_{1}$, the peaks can be shifted back and forth between right and left sidebands, and their widths correspond to the values of $\Gamma_{ \pm}$ and $\Gamma_{0}$ of dressed populations. Thus, assisted by the level spacing $\omega_{12}$, the interference induced by the anisotropic Purcell factor can not only control the width of emission peaks but also their spectral symmetry.

The Spontaneous Emission Spectrum in the Case of Subwavelength-Confined Anisotropic Purcell Factor. Among many available photonic nanostructures, ${ }^{39-43}$ plasmonic structures present large the anisotropy of the Purcell factor and enhanced local field, which enable nanoscalely varied quantum behavior of emission photons. ${ }^{33,34}$ So we chose plasmonic structures with the subwavelength confined anisotropic Purcell factor to demonstrate the above theory. The Green's tensor technique with a mesh of 20 to $25 \mathrm{~nm}$ to design the required plasmonic structures. ${ }^{51,52}$ Using current nanofabrication techniques, the designed plasmonic structure can be fabricated in the lab.

Although using nanoscale plasmonic structures to trap the atoms and control their motion is proposed, ${ }^{53,54}$ the accurate and stable positioning of the atoms remains challenging with present techniques. However, the above mechanism is the same as the three-level emitter with closely lying upper states. Hence, in the real experiments, quantum emitters can be replaced by the three-level quantum dot or nitrogen-vacancy center in diamond, ${ }^{55}$ whose precise positioning technique has been developed. $^{56-58}$

Here the assumption of closed atomic system is used, and coupling between its emission and surface plasmon modes is not discussed. ${ }^{59}$ When the coupling is included, efficient and directional generation of single photons ${ }^{17-19}$ and entanglement of two qubits ${ }^{35}$ have been reported.

The calculating parameters associated with the atomic system are discussed. This four-level system can be formed by the Rubidium 85 D2 hyperfine structure with the transition wavelength of $780 \mathrm{~nm}$. Two excited states $\left|a_{1}\right\rangle$ and $\left|a_{2}\right\rangle$, and two ground states $|b\rangle$ and $|c\rangle$ corresponds to $\left(5^{2} P_{3 / 2}, F=3\right)$ and $\left(5^{2} P_{3 / 2}, F=2\right)$, and $\left(5^{2} S_{1 / 2}, F=3\right)$ and $\left(5^{2} S_{1 / 2}, F=2\right)$, respectively. The decay rate of the upper state to ground states is $\Gamma=2 \pi^{*} 6 \mathrm{MHz}$ (or $\gamma_{0}=6 \mathrm{MHz}$ ). If we use a driving electric field with the value of $E=(5.0-10.0) \gamma_{0}$, the Rabi frequencies are $\Omega_{R}=(4.0-8.0) \gamma_{0}$, which can be fulfilled by the realistic experimental conditions.

Here, two dipoles are assumed to be orthogonal and their angle bisector is along the $z$ axis. In this case, the line width $\left(\Gamma_{0}\right.$ $\left.=2 \Gamma_{x x}, \Gamma_{ \pm}=\Gamma_{z z}\right)$ and the line width ratio $\left(\Gamma_{0} / \Gamma_{ \pm}=2 \Gamma_{x x} / \Gamma_{z z}\right)$ are directly determined by the anisotropy of the Purcell factor. In a vacuum, the line width ratio is 2 due to no interference. ${ }^{45}$ In the following, we will see that the spectral line width shows very fruitful behavior, that is, narrowing or widening, even the subnatural line width, in the case of subwavelength-confined anisotropic Purcell factor induced by the plasmon nanostructures.

Starting with a gold nanowire with a cross section of $50 \times 50$ $\mathrm{nm}^{2}$, we calculate the interference effects that arise from spontaneous emission spectra; the results are displayed in Figure 4. If the atom is too close or too far from the metallic structure, there is no obvious coherence effects due to fluorescence quenching ${ }^{60}$ or small anisotropy of the Purcell factor. With decreasing distance $d$, the anisotropy of the Purcell factor becomes large, but with large absolute values for $\Gamma_{z z}$ and $\Gamma_{x x}$. Until $d>150 \mathrm{~nm}$, these decay rates tend to the value $\gamma_{0}$ of a vacuum. If $d=50 \mathrm{~nm}$, the emission spectrum shows minimal
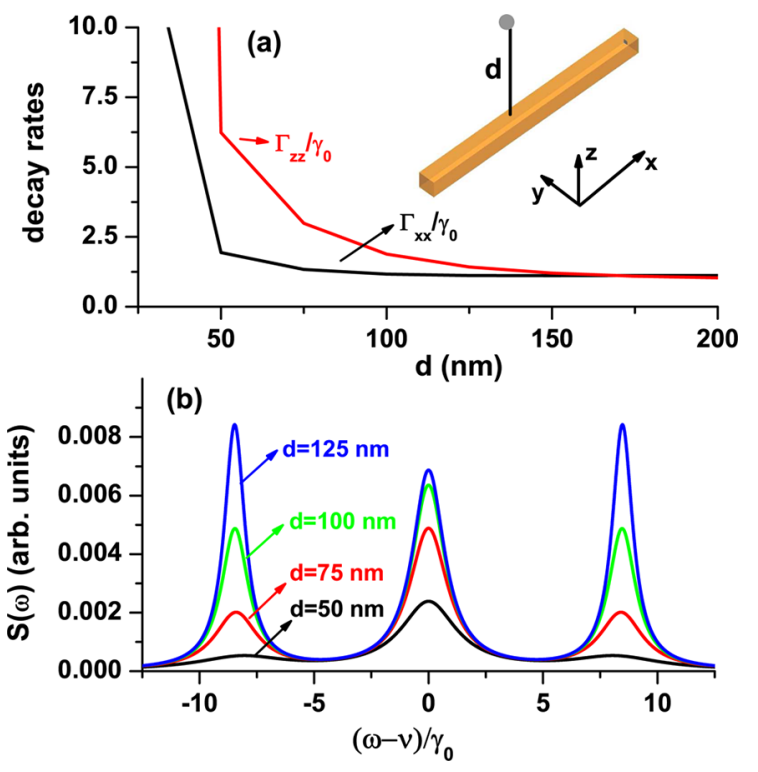

Figure 4. (a) Modified decay rates $\Gamma_{x x}$ and $\Gamma_{z z}$ as a function of distance $d$ from the metallic surface, and (b) spontaneous emission spectra of two orthogonal dipoles with varying $d$. The inset of (a) is the combined system of an atom and a gold nanowire. $\Omega_{1}=\Omega_{2}=6.0 \gamma_{0}$.

narrowing with $\Gamma_{0} / \Gamma_{ \pm}=0.6214$; at $d=75 \mathrm{~nm}$ and $d=100 \mathrm{~nm}$, this narrowing weakens from $\Gamma_{0} / \Gamma_{ \pm}=0.8948$ to $\Gamma_{0} / \Gamma_{ \pm}=1.236$ and almost disappears at $d=125 \mathrm{~nm}$ with $\Gamma_{0} / \Gamma_{ \pm}=1.572$. This weakening with distance can also be extended to metallic surfaces or metallic thin films where a similar anisotropy behavior of the Purcell factor occurs. ${ }^{16}$

We then propose a periodical gold nanostructure (see inset in Figure $5 \mathrm{~b}$ ), where the periodicity is $150 \mathrm{~nm}$ and the dimension of each cell is $100 \times 50 \times 50 \mathrm{~nm}^{3}$. Similar to nanowires, the anisotropy of the Purcell factor decreases with increasing distance between the atom and the metallic surface,
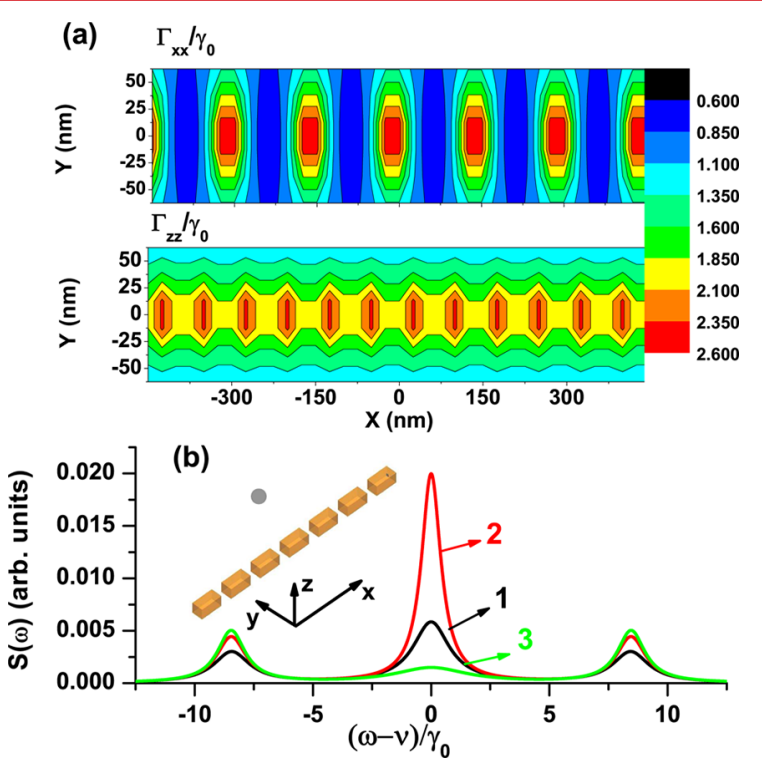

Figure 5. (a) Modified decay rates $\Gamma_{x x}$ and $\Gamma_{z z}$ over an $x y$-plane $50 \mathrm{~nm}$ away from a metallic surface, and (b) spontaneous emission spectra of two orthogonal dipoles at different positions (defined in the text). The inset of (b) is the combined system of an atom and a periodic gold nanostructure. $\Omega_{1}=\Omega_{2}=6.0 \gamma_{0}$. 
resulting in weaker interference. However, distinct from nanowires, decay rates $\Gamma_{z z}$ and $\Gamma_{x x}$ are distributed periodically. On the $x y$-plane $50 \mathrm{~nm}$ from the metallic surface, as shown in Figure 5a, $\Gamma_{z z}>\Gamma_{x x}$ at point 1 (at the edge of the cell) and point 2 (in the middle), but a larger anisotropy is observed in its middle part; between the gaps of two cells (point 3 ), $\Gamma_{z z}<$ $\Gamma_{x x}$. The periodically distributed decay rates are reflected in the emission spectra as narrowing of the central line width at point 1 with $\Gamma_{0} / \Gamma_{ \pm}=1.009$, via a line width minimum at point 2 with $\Gamma_{0} / \Gamma_{ \pm}=0.6573$, and finally broadening at point 3 with $\Gamma_{0} / \Gamma_{ \pm}=$ 2.650. Thus, a periodical anisotropic Purcell factor induced by structural periodicity can modify the spontaneous emission spectrum periodically.

The above structures only provide large anisotropy of the Purcell factor, but absolute values of decay rates are very large, which prevent further narrowing of spectral lines. The customdesigned resonant plasmon nanotructure is composed of 14 gold nanostrips of $140 \times 60 \times 40 \mathrm{~nm}^{3}$ with $40 \mathrm{~nm}$ spacing in both directions and resonances at the wavelength of $780 \mathrm{~nm}$ (Figure 6a). The interleaving arrangement not only guarantees

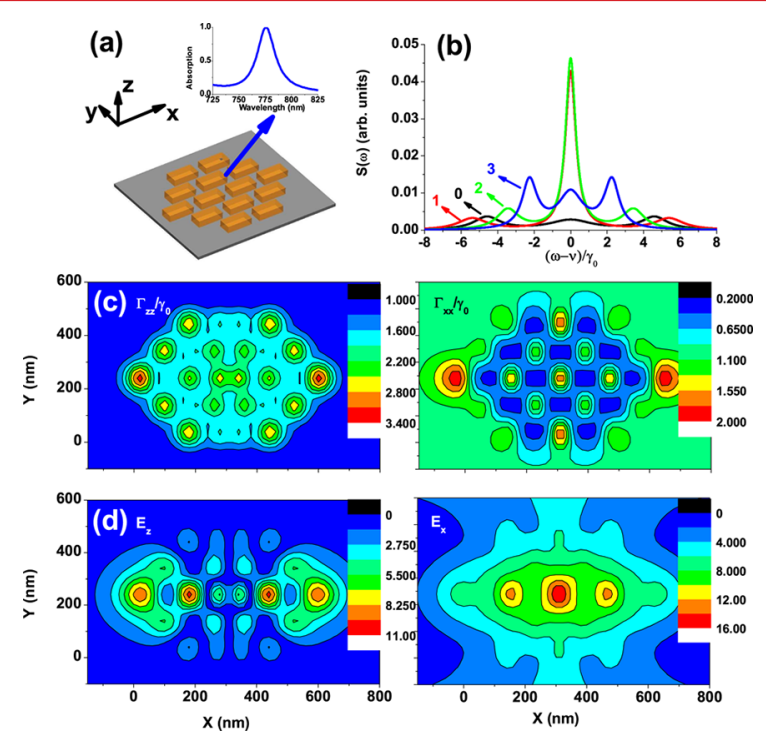

Figure 6. (a) Schematic of a resonant gold nanostructure to excite atoms at wavelength $\lambda=780 \mathrm{~nm}$, (b) modified spontaneous emission spectra of two orthogonal dipoles at points $0,1,2$, and 3 (defined in the text), and (c) modified decay rates $\Gamma_{z z}$ and $\Gamma_{x x}$ and (d) near field distributions $E_{z}$ and $E_{x}$ on the $x y$-plane $60 \mathrm{~nm}$ from the metallic surface.

large anisotropy of the Purcell factor with relatively small decay rates but also strong near field at resonance (Figures $6 c, d$ ). It is known that large anisotropy of the Purcell factor only exists at about $30-120 \mathrm{~nm}$ nanoregions around the metallic nanostructures. ${ }^{33}$ When single atoms are placed within the $x y$-plane 40 $\mathrm{nm}$ from the gold surface, significant interference effects in the spontaneous spectrum are observed. Here, the electric fields associated with Rabi frequencies is normalized by 2 and the center point or point 0 is at $(0,0) \mathrm{nm}$. There is a subnatural narrowing of the spectral lines associated with the central emission peak for points 1 (at $(100,0) \mathrm{nm}$ ) and 2 (at $(200,0)$ $\mathrm{nm}$ ) (Figure 6b)) for $\Gamma_{z z}$ is several times of $\Gamma_{x x}$ and $2 \Gamma_{x x}<1$. Not a strong narrowing is observed for point 0 or point 3 (at $(0,100) \mathrm{nm})$ due to small anisotropy of the Purcell factor or weak Rabi frequency. The sidebands are very sensitive to the atomic positions due to different local field excitations. Thus, the nanoscale anisotropic Purcell factor within a resonant plasmonic structure can dramatically modify spontaneous emission spectra.

In summary, using the dressed-state analysis and quantum regression theorem, we have theoretically demonstrated a mechanism exploiting the anisotropic Purcell factor to control the linewidths of atomic spontaneous emissions. As proof of the mechanism and, in particular, its application in the case of the subwavelength-confined anisotropic Purcell factor, several plasmonic structures were employed and interesting phenomena in the spontaneous emission spectrum appear, for example, atomic spectral line rapid narrowing, nanoscale line width pulsing, and dramatic modification, in particular, subnatural line width for two orthogonal dipoles, at the nanoscale. The study bridges the fields of quantum optics and plasmonics associated with the ultrasmall optical mode area and will benefit for lowlevel light nonlinear optical properties ${ }^{2-5}$ and efficient coupling of single photons into the single plasmons. ${ }^{17-19,61-63}$ The present protocol assisted by the plasmon nanostructure is propitious to implement quantum interferences experimentally in the spontaneous emission of quantum emitters with closely lying upper states. Superior to the cavity QED, plasmoninduced anisotropic vacuum and plasmon excitation cover a broad frequency region and require no sophisticated experimental setups to achieve the resonances. Thus, the combined system of quantum emitters and plasmon nanostructure may open some perspectives for applications in ultracompact active quantum devices.

\section{ASSOCIATED CONTENT}

\section{Supporting Information}

The equations of motion of the reduced density matrix elements for the atomic variables, which explicitly include the decay rates of $\rho_{00}$ and $\rho_{++}$, have been provided. This material is available free of charge via the Internet at http://pubs.acs.org.

\section{AUTHOR INFORMATION}

\section{Corresponding Author}

*E-mail: (Y.G.)ygu@pku.edu.cn; (Q.G.) qhgong@pku.edu.cn.

\section{Notes}

The authors declare no competing financial interest.

\section{ACKNOWLEDGMENTS}

This work was supported by the National Natural Science Foundation of China under Grants 91121018 and 11121091 and National Key Basic Research Program under Grant 2007CB307001.

\section{REFERENCES}

(1) Brongersma, M. L.; Shalaev, V. M. Science 2010, 328, 440.

(2) Ghosh, S.; Bhagwat, A. R.; Renshaw, C. K.; Goh, S.; Gaeta, A. L. Phys. Rev. Lett. 2006, 97, 023603.

(3) Spillane, S. M.; Pati, G. S.; Salit, K.; Hall, M.; Kumar, P.; Beausoleil, R. G.; Shahriar, M. S. Phys. Rev. Lett. 2008, 100, 233602.

(4) Londero, P.; Venkataraman, V.; Bhagwat, A. R.; Slepkov, A. D.; Gaeta, A. L. Phys. Rev. Lett. 2009, 103, 043602.

(5) Hendrickson, S. M.; Lai, M. M.; Pittman, T. B.; Franson, J. D. Phys. Rev. Lett. 2010, 105, 173602.

(6) Kuttge, M.; de Abajo, F. J. G.; Polman, A. Nano Lett. 2010, 10, $1537-1541$.

(7) Savasta, S.; Saija, R.; Ridolfo, A.; Stefano, O. D.; Denti, P.; Borghese, F. ACS Nano 2010, 4, 6369-6376. 
(8) Passmore, B. S.; Adams, D. C.; Ribaudo, T.; Wasserman, D.; Lyon, S.; Davids, P.; Chow, W. W.; Shaner, E. A. Nano Lett. 2011, 11, 338-342.

(9) Artuso, R. D.; Bryant, G. W. Nano Lett. 2008, 8, 2106-2111.

(10) Fofang, N. T.; Grady, N. K.; Fan, Z.; Govorov, A. O.; Halas, N. J. Nano Lett. 2011, 11, 1556-1560.

(11) Manjavacas, A.; de Abajo, F. J. G.; Nordlander, P. Nano Lett. 2011, 11, 2318-2323.

(12) Zhang, W.; Govorov, A. O.; Bryant, G. W. Phys. Rev. Lett. 2006, 97, 146804.

(13) Ridolfo, A.; Stefano, O. D.; Fina, N.; Saija, R.; Savasta, S. Phys. Rev. Lett. 2010, 105, 263601.

(14) Purcell, E. M. Phys. Rev. 1946, 69, 681.

(15) Andersen, M. L.; Stobbe, S.; Sorensen, A. S.; Lodahl, P. Nat. Phys. 2011, 7, 215.

(16) Chance, R. R.; Prock, A.; Silbey, R. J. Chem. Phys. 1975, 62, 2245-2253.

(17) Akimov, A. V.; Mukherjee, A.; Yu, C. L.; Chang, D. E.; Zibrov, A. S.; Hemmer, P. R.; Park, H.; Lukin, M. D. Nature 2007, 450, 402406.

(18) Chang, D. E.; Sørensen, A. S.; Hemmer, P. R.; Lukin, M. D. Phys. Rev. Lett. 2006, 97, 053002.

(19) Chang, D. E.; Sørensen, A. S.; Demler, E. A.; Lukin, M. D. Nat. Phys. 2007, 3, 807-812.

(20) Wokaun, A.; Gordon, J. P.; Liao, P. F. Phys. Rev. Lett. 1982, 48, 957-960.

(21) Lal, S.; Link, S.; Halas, N. J. Nat. Photonics 2007, 1, 641-648.

(22) Wokaun, A.; Bergman, J. G.; Heritage, J. P.; Glass, A. M.; Liao, P. F.; Olson, D. H. Phys. Rev. B 1981, 24, 849-856.

(23) Kim, E. M.; Elovikov, S. S.; Murzina, T. V.; Nikulin, A. A.; Aktsipetrov, O. A.; Bader, M. A.; Marowsky, G. Phys. Rev. Lett. 2005, 95, 227402.

(24) Danckwerts, M.; Novotny, L. Phys. Rev. Lett. 2007, 98, 026104.

(25) Kulkarni, A. P.; Noone, K. M.; Munechika, K.; Guyer, S. R.; Ginger, D. S. Nano Lett. 2010, 10, 1501-1505.

(26) Derkacs, D.; Lim, S. H.; Matheu, P.; Mar, W.; Yu, E. T. Appl. Phys. Lett. 2006, 89, 093103.

(27) Lakowicz, J. R. Plasmonics 2006, 1, 5-33.

(28) Anger, P.; Bharadwaj, P.; Novotny, L. Phys. Rev. Lett. 2006, 96, 113002.

(29) Kühn, S.; Håkanson, U.; Rogobete, L.; Sandoghdar, V. Phys. Rev. Lett. 2006, 97, 017402.

(30) Bharadwaj, P.; Novotny, L. Opt. Express 2007, 15, 1426614274

(31) Kulakovich, O.; Strekal, N.; Yaroshevich, A.; Maskevich, S.; Gaponenko, S.; Nabiev, I.; Woggon, U.; Artemyev, M. Nano Lett. 2002, 2, 1449-1452.

(32) Tam, F.; Goodrich, G. P.; Johnson, B. R.; Halas, N. J. Nano Lett. 2007, 7, 496-501.

(33) Gu, Y.; Huang, L.; Martin, O. J. F.; Gong, Q. Phys. Rev. B 2010, $81,193103$.

(34) Marty, R.; Arbouet, A.; Paillard, V.; Girard, C.; des Francs, G. C. Phys. Rev. B 2010, 82, 081403(R).

(35) Gonzalez-Tudela, A.; Martin-Cano, D.; Moreno, E.; MartinMoreno, L.; Tejedor, C.; Garcia-Vidal, F. J. Phys. Rev. Lett. 2011, 106, 020501.

(36) Yang, W.; Conkey, D. B.; Wu, B.; Yin, D.; Hawkins, A. R.; Schmidt, H. Nat. Photonics 2007, 1, 331-335.

(37) Zhou, P.; Swain, S. Phys. Rev. Lett. 1996, 77, 3995-3998.

(38) Paspalakis, E.; Knight, P. L. Phys. Rev. Lett. 1998, 81, 293-296.

(39) Quang, T.; Woldeyohannes, M.; John, S.; Agarwal, G. S. Phys. Rev. Lett. 1997, 79, 5238-5241.

(40) Zhu, S.-Y.; Chen, H.; Huang, H. Phys. Rev. Lett. 1997, 79, 205208.

(41) Li, G.-X.; Li, F.-L.; Zhu, S.-Y. Phys. Rev. A 2001, 64, 013819.

(42) Yang, Y.; Xu, J.; Chen, H.; Zhu, S. Phys. Rev. Lett. 2008, 100, 043601.

(43) Yannopapas, V.; Paspalakis, E.; Vitanov, N. V. Phys. Rev. Lett. 2009, 103, 063602.
(44) Agarwal, G. S. Phys. Rev. Lett. 2000, 84, 5500-5503.

(45) Zhu, S.-Y.; Scully, M. O. Phys. Rev. Lett. 1996, 76, 388-391.

(46) Lee, H.; Polynkin, P.; Scully, M. O.; Zhu, S.-Y. Phys. Rev. A 1997, 55, 4454-4465.

(47) Scully, M. O., Zubairy, M. S., Eds. Quantum optics; Combridge University Press: Cambridge, 1997.

(48) Barnett, S. M.; Huttner, B.; Loudon, R.; Matloob, R. J. Phys. B 1996, 29, 3763-3781.

(49) Cohen-Tannoudji, C.; Reynaud, S. J. Phys. B 1977, 10, 345-363.

(50) Gu, Y.; Wang, L.; Ren, P.; Zhang, J.; Zhang, T.; Xu, J.-P.; Zhu, S.-Y.; Gong, Q. Plasmonics 2012, 7, 33-38.

(51) Gu, Y.; Chen, L.; Zhang, H.; Gong, Q. EPL 2008, 83, 27004.

(52) Martin, O. J. F.; Girard, C.; Dereux, A. Phys. Rev. Lett. 1995, 74, 526-529.

(53) Chang, D. E.; Thompson, J. D.; Park, H.; Vuletić, V.; Zibrov, A. S.; Zoller, P.; Lukin, M. D. Phys. Rev. Lett. 2009, 103, 123004.

(54) Stehle, C.; Bender, H.; Zimmermann, C.; Kern, D.; Fleischer, M.; Slama, S. Nat. Photonics 2011, 5, 494-498.

(55) Santori, C.; Fattal, D.; Spillane, S. M.; Fiorentino, M.; Beausoleil, R. G.; Greentree, A. D.; Olivero, P.; Draganski, M.; Rabeau, J. R.; Reichart, P.; Gibson, B. C.; Rubanov, S.; Jamieson, D. N.; Prawer, S. Opt. Express 2006, 14, 7986-7993.

(56) Badolato, A.; Hennessy, K.; Atatüre, M.; Dreiser, J.; Hu, E.; Petroff, P. M.; Imamoğlu, A. Science 2005, 308, 1158-1161.

(57) van der Sar, T.; Heeres, E. C.; Dmochowski, G. M.; de Lange, G.; Robledo, L.; Oosterkamp, T. H.; Hanson, R. Appl. Phys. Lett. 2009, 94, 173104

(58) Englund, D.; Shields, B.; Rivoire, K.; Hatami, F.; Vučković, J.; Park, H.; Lukin, M. D. Nano Lett. 2010, 10, 3922-3926.

(59) Strong coupling between atoms and single surface plasmons is very important in the generation of single plasmons source, which will be further investigated in our other publications.

(60) Dulkeith, E.; Morteani, A. C.; Niedereichholz, T.; Klar, T. A.; Feldmann, J.; Levi, S. A.; van Veggel, F. C. J. M.; Reinhoudt, D. N.; Möller, M.; Gittins, D. I. Phys. Rev. Lett. 2002, 89, 203002.

(61) Fedutik, Y.; Temnov, V. V.; Schöps, O.; Woggon, U.; Artemyev, M. V. Phys. Rev. Lett. 2007, 99, 136802.

(62) Tame, M. S.; Lee, C.; Lee, J.; Ballester, D.; Paternostro, M.; Zayats, A. V.; Kim, M. S. Phys. Rev. Lett. 2008, 101, 190504.

(63) Huck, A.; Smolka, S.; Lodahl, P.; Sørensen, A. S.; Boltasseva, A.; Janousek, J.; Andersen, U. L. Phys. Rev. Lett. 2009, 102, 246802. 